\title{
Cyclic energy pathways in ecological food webs
}

\author{
Brian D. Fath ${ }^{a, b, *}$, Geir Halnes ${ }^{b, c}$ \\ a Biology Department, Towson University, Towson, MD 21252, USA \\ b Dynamic Systems Project, International Institute for Applied Systems Analysis, Laxenburg, Austria \\ c Swedish University of Agricultural Sciences, PO Box 7070, SE-750 07 Uppsala, Sweden
}

\section{A R T I C L E I N F O}

\section{Article history:}

Accepted 26 April 2007

Published on line 13 June 2007

Keywords:

Cycling

Detritus

Energy flow

Food webs

Network analysis

Trophic dynamics

\begin{abstract}
A B S T R A C T
Standard ecology textbooks typically maintain that nutrients cycle, but energy flows in unidirectional chains. However, here we use a new metric that allows for the identification and quantification of cyclic energy pathways. Some of these important pathways occur due to the contribution of dead organic matter to detrital pools and those organisms that feed on them, reintroducing some of that energy back into the food web. Recognition of these cyclic energy pathways profoundly impacts many aspects of ecology such as trophic levels, control, and the importance of indirect effects. Network analysis, specifically the maximum eigenvalue of the connectance matrix, is used to identify both the presence and strength of these structural cycles.
\end{abstract}

(c) 2007 Elsevier B.V. All rights reserved.
"Following death, every organism is a potential source of energy for saprophagous organisms (feeding directly on dead tissue), which again may act as energy sources for successive categories of consumers".

Lindeman (1942, p. 400).

\section{Introduction}

It is clear from both Lindeman's original diagram and text that he envisioned energy flow pathways as cyclical. However, in 1942, Lindeman did not have the methodological tools to simultaneously investigate the complex conceptual network that he used to represent the energy pathways in the Cedar Bog Lake ecosystem, and therefore, out of practicality, reduced the analysis to a series of two parallel trophic chains. As a result, food web ecology has developed largely along this paradigm in which matter-energy flow transfers mostly sequentially from basal to intermediate to top species (e.g., Paine, 1980; Cohen et al., 1990; Pimm, 2002). Some exceptions were evident, especially in marine ecosystems (e.g., Pomeroy, 1974; Wiegert and
Owen, 1971; Ulanowicz, 1983; Wetzel, 1995, and notably in a related series of papers called network trophic dynamics literature, e.g., Patten, 1985; Burns, 1989; Burns et al., 1991; Higashi et al., 1988, 1991, 1993a,b; Patten et al., 1990; Whipple and Patten, 1993; Whipple, 1998, 1999). In flow analysis of food webs, cycling affects, for instance, residence time and the total system throughflow (Fath et al., 2004). For example, Patten (1985) used network flow analysis to show energy is available and cycling in the Okefenokee Swamp ecosystem (some "trophic" transfers were over 20 steps in length). Recently, this detrital pathway has recently garnered renewed attention within the ecological community (see Moore et al., 2004), but the data requirements for a flow analysis similar to Patten (1985) are great which has impeded the application of this methodology to many systems. In fact, most published food webs or models have structural data (i.e., connectance), but not functional data (flow or interaction strength). Therefore, it is useful to have a metric that identifies the cyclic pathways based only on the connectance pattern. This paper adds to the network trophic dynamics paradigm some novel results

\footnotetext{
* Corresponding author at: Biology Department, Towson University, Towson, MD 21252, USA. Tel.: +1 4107042535 ; fax: +1 4107042405.

E-mail address: bfath@towson.edu (B.D. Fath).
} 
for structural networks that are under growing discussion in recent literature.

A structural cycle is the presence of a pathway in the ecological network in which matter-energy passes through biotic or abiotic stores returning for availability to the same or lower trophic levels. Structural cycling is present in food webs due to intraguild predation, cannibalism, or other predation events that connect laterally or backwards in the hierarchy. Many empirical food web descriptions capture these cycles, and also assembly models such as the Constant Connectance (Martinez, 1992) and Niche model (Williams and Martinez, 2000) allow for internal structural cycles. Common to these models and many empirical data sets, is that all the structural cycling is due to "traditional" predation, and that they ignore the extra feedback connections caused by flows to detritus and back to the system through detritus feeders. Flows to detritus include many pathways such as death, excrement, or exfoliation. The detritus feedback loop is a fixed cyclic structure. It assures a certain amount of cycling, and it is fundamentally different from other cyclic structures in the system since it allows energy to flow from any trophic level including top predators to lower trophic levels.

In this paper we investigate 26 empirically derived ecological food webs with and without detrital interconnections ranging in size from 6 to 220 compartments to demonstrate the importance of the detritus feedback loop and we compare the results to the structural cycling generated by five different community assembly rules.

\section{Structural cycling}

Common network analysis properties include network size (n) and connectance (C). Connectance is defined as $C=L / n^{2}$, where $L$ is the number of links and $n^{2}$ is the possible number of connections in the network. Furthermore, $n C=L / n$, which is the property known as linkage density. A newly developed and important property is network structural cycling (Fath, 1998; Jain and Krishna, 2003). This measures the presence and strength of cyclic pathways in a strongly connected component (SCC) of a network. There has not been much application of SCC to ecosystems, but Allesina et al. (2005a) recently demonstrated how one could decompose food webs into SCC to determine possible compartments. For an irreducible matrix, $\lambda_{\max }$ is bounded by $n$. For a reducable matrix (several SCCs), $\lambda_{\max }$ is bounded by the number of nodes in the largest subcomponent, and is the dominant eigenvalue for the subcomponent with the strongest degree of structural cycling. All food webs studied here with the detrital loop were irreducible (\# of SCC =1). The eigenvalue does not measure the quantity of flow; and therefore, differs from the Finn (1976) cycling index, which is a measure of cycled flow.

The strength of structural cycling or cyclicity is given by the magnitude of the largest eigenvalue (also called the spectral radius), $\lambda_{\max }$ of the structural adjacency matrix. A structural connectance matrix, or adjacency matrix, $A$, is a binary representation of the connections such that $a_{i j}=1$ if there is (a)

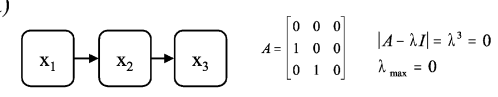

(b)

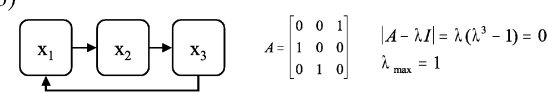

(c)

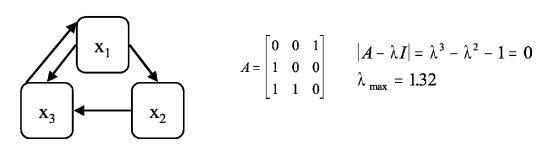

Fig. 1 - Examples of structures which exhibit the three different types of cycling based on eigenvalue analysis. (a) No cycling: $\lambda_{\max }=0$, (b) weak cycling: $\lambda_{\max }=1$, and (c) strong cycling: $\lambda_{\max }>1$.

a connection from $j$ to $i$, and a zero otherwise. ${ }^{1}$ There are three possibilities for the spectral radius: $\lambda_{\max }=0, \lambda_{\max }=1$, and $\lambda_{\max }>1$, respectively (Fig. 1) (Fath, 1998; Jain and Krishna, 2003). When $\lambda_{\max }=0$, then all eigenvalues equal zero and there is no structural cycling and no indirect pathways greater in length than $n-1$; weak structural cycling occurs when $\lambda_{\max }=1$, indicating the presence of cycles, but the number of pathways between nodes does not increase geometrically; lastly, when $\lambda_{\max }>1$, strong structural cycling occurs, in which case the number of pathways between two compartments increases without bound at a constant rate equal to $\lambda_{\max }$ as the path length between those two compartments increases. For example, Lindeman's Cedar Bog Lake food web (which he called a food-cycle) has eight compartments, including two primary producers: (1) phytoplankton and (2) pond weeds; two grazers: (3) zooplankton and (4) browsers; three predators: (5) plankton predators, (6) benthic predators and (7) swimming predators, all of which are connected to a central exchange compartment labeled (8) ooze and bacteria, which clearly represents detritus and detrital feeders. In the corresponding adjacency matrix, $a_{83}=1$ indicates there is one direct path from zooplankton $(j=3)$ to ooze $(i=8)$.

$A=\left[\begin{array}{llllllll}0 & 0 & 0 & 0 & 0 & 0 & 0 & 0 \\ 0 & 0 & 0 & 0 & 0 & 0 & 0 & 0 \\ 1 & 0 & 0 & 0 & 0 & 0 & 0 & 1 \\ 0 & 1 & 0 & 0 & 0 & 0 & 0 & 1 \\ 1 & 0 & 1 & 0 & 0 & 0 & 0 & 1 \\ 0 & 1 & 0 & 1 & 0 & 0 & 0 & 1 \\ 1 & 1 & 1 & 1 & 1 & 1 & 0 & 0 \\ 1 & 1 & 1 & 1 & 1 & 1 & 1 & 0\end{array}\right]$,

$n=8, \quad C=\frac{23}{64}=0.359, \quad n C=2.87, \quad \lambda_{\max }=2.58$

Furthermore, using matrix multiplication, $A^{m}$ gives the number of pathways between compartments of length $m$, such that

\footnotetext{
${ }^{1}$ Note the orientation of flow from $j$ to $i$ is used because that makes the direction of ecological relation from $i$ to $j$. For example, if $i$ preys on $j$, the flow of energy is from $j$ to $i$.
} 
$A^{2}$ shows there are two pathways of length 2 from zooplankton to ooze (zooplankton $\rightarrow$ plankton predators $\rightarrow$ ooze and zooplankton $\rightarrow$ swimming predators $\rightarrow$ ooze), and $A^{3}$ that there are five pathways of length 3 , etc., which for this system are given as:

$A^{2}=\left[\begin{array}{llllllll}0 & 0 & 0 & 0 & 0 & 0 & 0 & 0 \\ 0 & 0 & 0 & 0 & 0 & 0 & 0 & 0 \\ 1 & 1 & 1 & 1 & 1 & 1 & 1 & 0 \\ 1 & 1 & 1 & 1 & 1 & 1 & 1 & 0 \\ 2 & 1 & 1 & 1 & 1 & 1 & 1 & 1 \\ 1 & 2 & 1 & 1 & 1 & 1 & 1 & 1 \\ 2 & 2 & 1 & 1 & 0 & 0 & 0 & 4 \\ 3 & 3 & 2 & 2 & 1 & 1 & 0 & 4\end{array}\right] ;$

$A^{3}=\left[\begin{array}{llllllll}0 & 0 & 0 & 0 & 0 & 0 & 0 & 0 \\ 0 & 0 & 0 & 0 & 0 & 0 & 0 & 0 \\ 3 & 3 & 2 & 2 & 1 & 1 & 0 & 4 \\ 3 & 3 & 2 & 2 & 1 & 1 & 0 & 4 \\ 4 & 4 & 3 & 3 & 2 & 2 & 1 & 4 \\ 4 & 4 & 3 & 3 & 2 & 2 & 1 & 4 \\ 5 & 5 & 4 & 4 & 4 & 4 & 4 & 2 \\ 7 & 7 & 5 & 5 & 4 & 4 & 4 & 6\end{array}\right]$.

Note, not only do the number of pathways increase as path length increases, but also in the limit as $m \rightarrow \infty$, they increase at a rate equal to the spectral radius: $a_{i j}^{(m+1)} / a_{i j}^{(m)} \rightarrow \lambda \max$ (Seneta, 1973; Fath, 1998; Hill, personal communication). For Cedar Bog Lake, $\lambda_{\max }=2.58$, so there is strong structural cycling. Cycling is a key feature of the web. It indicates an increasing number of pathways for material and energy transfer and this feedback provides a tightly coupled dependence between species.

\section{Data sources}

In a well-studied set of 17 food webs (Dunne et al., 2002, 2004), 10 of the webs explicitly include detritus compartments as source compartments (out-degree greater than zero), but with no input (in-degree equal to zero) ${ }^{2}$; the other seven webs excluded detritus entirely. This gives the image of two parallel food chains, one starting from primary producers - whose energy source is solar radiation - and the other beginning with a detrital pool, which has neither internal nor external input, thereby acting as an infinite energy source. A more realistic web (e.g., Wiegert and Owen, 1971) has the detritus receiving input from the other within system compartments or external input, thereby ensuring detritus has an in-degree of greater than one and maintaining mass balance.

In this paper we examine the 10 empirically derived food webs from Dunne et al. (2002) (Polis, 1991; Goldwasser and

\footnotetext{
${ }^{2}$ In-degree and out-degree are standard structural network metrics that give the number of arcs terminating or emanating from a particular node, respectively.
}

Roughgarden, 1993; Waide and Reagan, 1996; Warren, 1989; Havens, 1992; Townsend et al., 1998; Christian and Luczkovich, 1999; Opitz, 1996; Link, 2002) that include detritus in the data sets but with no input into that compartment (set E1), and 16 empirically derived food webs (Lindeman, 1942; Heymans et al., 2002; Sandberg et al., 2000; Dame and Patten, 1981; Patten, 1988; Leguerrier et al., 2003; Patricio et al., 2004) that have the detritus feedback loop explicitly included (set E2). We compare the values of $\lambda_{\max }$ in these two datasets as well as in the first dataset modified to include detrital links. Lastly, we compare these empirical datasets with the results produced by five community assembly models with or without detritus.

\section{Community assembly rules}

Due to the difficulty in acquiring the requisite data for fully developed empirical food webs, researchers have developed community assembly rules that recreate, as best as possible, the perceived structural characteristics of food webs. These provided null-models to investigate fundamental questions of community structure and organization, attempting to link the ecological observed process to underlying pattern. Here we consider five such models, Constant Connectance (Martinez, 1992), Cascade (Cohen and Newman, 1985), Niche (Williams and Martinez, 2000), Modified Niche (Halnes et al., 2007), and Cyber-Ecosystem (Fath, 2004a). In the Cascade model, the $n$ species are ranked from 1 to $n$ and all species predate on a random number of the species with lower rank than themselves, thus establishing a strict series of food chains. In the Constant Connectance model or random model, all the $n$ species are connected randomly among each other, with each connection having the same probability of occurring. A criticism of the random model is that it has no biological or ecological constraints incorporated in the structural principles. The Niche model allows for a limited amount of cycling but only within a small range around each species, called the niche-interval. It does not explicitly include flows to or from detritus. The Modified Niche model follows the basic rules of the Niche model but adds an additional detritus compartment, and includes outflow from each compartment to detritus, and flow back into the system through the probability of feeding on detritus. This fixes the mass balance error of the original Niche model and adds an extra structure that is fundamentally different from other structures since it includes transfers from top predators to lower trophic levels. The Cyber-Ecosystem model uses a meta-structure of six functional groups: primary producers, herbivores, carnivores, omnivores, detritus and detrital feeders, within which random connections within functional groups link species based on these definitional constraints.

\section{Results}

The maximum eigenvalue was calculated using the data for the empirical food webs without detritus (E1), in which $n$ ranges from 30 to 220 (Table 1 ). The data have relatively low $\lambda_{\max }$ (range from 0 to 10.25) and no readily apparent pattern regarding structural cycling (Fig. 2a). However, as stated above, although the webs include detritus in the categorical descrip- 
Table 1 - Empirical food webs representing aquatic and terrestrial ecosystems ranging from 6 to 220 components

\begin{tabular}{|c|c|c|c|c|c|c|c|c|}
\hline Ecosystem & Habitat & $n$ & $\begin{array}{c}n C \\
\text { (no detritus) }\end{array}$ & $\begin{array}{c}n C \\
\text { (with detritus) }\end{array}$ & $\begin{array}{c}\lambda_{\max } \\
\text { (no detritus) }\end{array}$ & $\begin{array}{c}\lambda_{\max } \\
\text { (detritus) }\end{array}$ & $\begin{array}{c}\text { \#SCC } \\
\text { (original }^{\mathrm{a}} \text { ) }\end{array}$ & Reference \\
\hline Canton Creek & Stream & 109 & 6.50 & 7.50 & 1.00 & 7.83 & 2 & Townsend et al. (1998) \\
\hline Stony Creek & Stream & 113 & 7.36 & 8.36 & 1.00 & 7.79 & 2 & Townsend et al. (1998) \\
\hline St. Marks Estuary & Estuary & 48 & 4.60 & 5.60 & 1.00 & 6.83 & 1 & Christian and Luczkovich (1999) \\
\hline Caribbean Reef small & Marine & 50 & 11.12 & 12.12 & 8.63 & 11.96 & 1 & Opitz (1996) \\
\hline NE US Shelf & Marine & 81 & 18.31 & 19.31 & 4.87 & 12.93 & 1 & Link (2002) \\
\hline Bridge Brook Lake & Lake/pond & 220 & 2.51 & 3.51 & 4.00 & 4.00 & 146 & Havens (1992) \\
\hline Skipworth Pond & Lake/pond & 37 & 2.62 & 3.62 & 2.00 & 5.77 & 3 & Warren (1989) \\
\hline St. Martin Island & Terrestrial & 44 & 3.00 & 4.00 & 0 & 5.27 & 2 & Goldwasser and Roughgarden (1993) \\
\hline Coachella Valley & Terrestrial & 30 & 2.13 & 3.13 & 1.00 & 1.62 & 1 & Polis (1991) \\
\hline El Verde Rainforest & Terrestrial & 156 & 9.68 & 10.68 & 10.25 & 12.17 & 1 & Waide and Reagan (1996) \\
\hline Brouage & Estuary & 13 & & 2.54 & & 1.79 & 1 & Leguerrier et al. (2003) \\
\hline Graminoids (wet season) & Estuary & 66 & & 12.02 & & 11.06 & 1 & Heymans et al. (2002) \\
\hline Graminoids (dry season) & Estuary & 66 & & 12.02 & & 11.06 & 1 & Heymans et al. (2002) \\
\hline Cypress (wet season) & Estuary & 68 & & 8.01 & & 6.85 & 1 & Heymans et al. (2002) \\
\hline Cypress (wet season) & Estuary & 68 & & 8.15 & & 7.06 & 1 & Heymans et al. (2002) \\
\hline Mangrove (wet season) & Estuary & 94 & & 14.26 & & 14.16 & 1 & Heymans et al. (2002) \\
\hline Mangrove (dry season) & Estuary & 94 & & 14.24 & & 14.17 & 1 & Heymans et al. (2002) \\
\hline Florida Bay (wet season) & Estuary & 125 & & 14.50 & & 10.97 & 1 & Heymans et al. (2002) \\
\hline Florida Bay (dry season) & Estuary & 125 & & 15.75 & & 11.01 & 1 & Heymans et al. (2002) \\
\hline Baltic Proper & Marine & 12 & & 3.00 & & 3.10 & 1 & Sandberg et al. (2000) \\
\hline Bothnian Sea & Marine & 12 & & 2.83 & & 2.81 & 1 & Sandberg et al. (2000) \\
\hline Bothnian Bay & Marine & 12 & & 2.67 & & 2.68 & 1 & Sandberg et al. (2000) \\
\hline Oyster Reef & Marine & 6 & & 2.00 & & 2.15 & 1 & Dame and Patten (1981) \\
\hline Okefenokee Swamp & Lake/pond & 24 & & 4.83 & & 5.08 & 1 & Patten et al. (1989) \\
\hline Zostera meadows & Lake/pond & 28 & & 6.18 & & 6.98 & 1 & Patricio et al. (2004) \\
\hline Cedar Bog Lake & Lake/pond & 8 & & 2.88 & & 2.58 & 1 & Lindeman (1942) \\
\hline
\end{tabular}



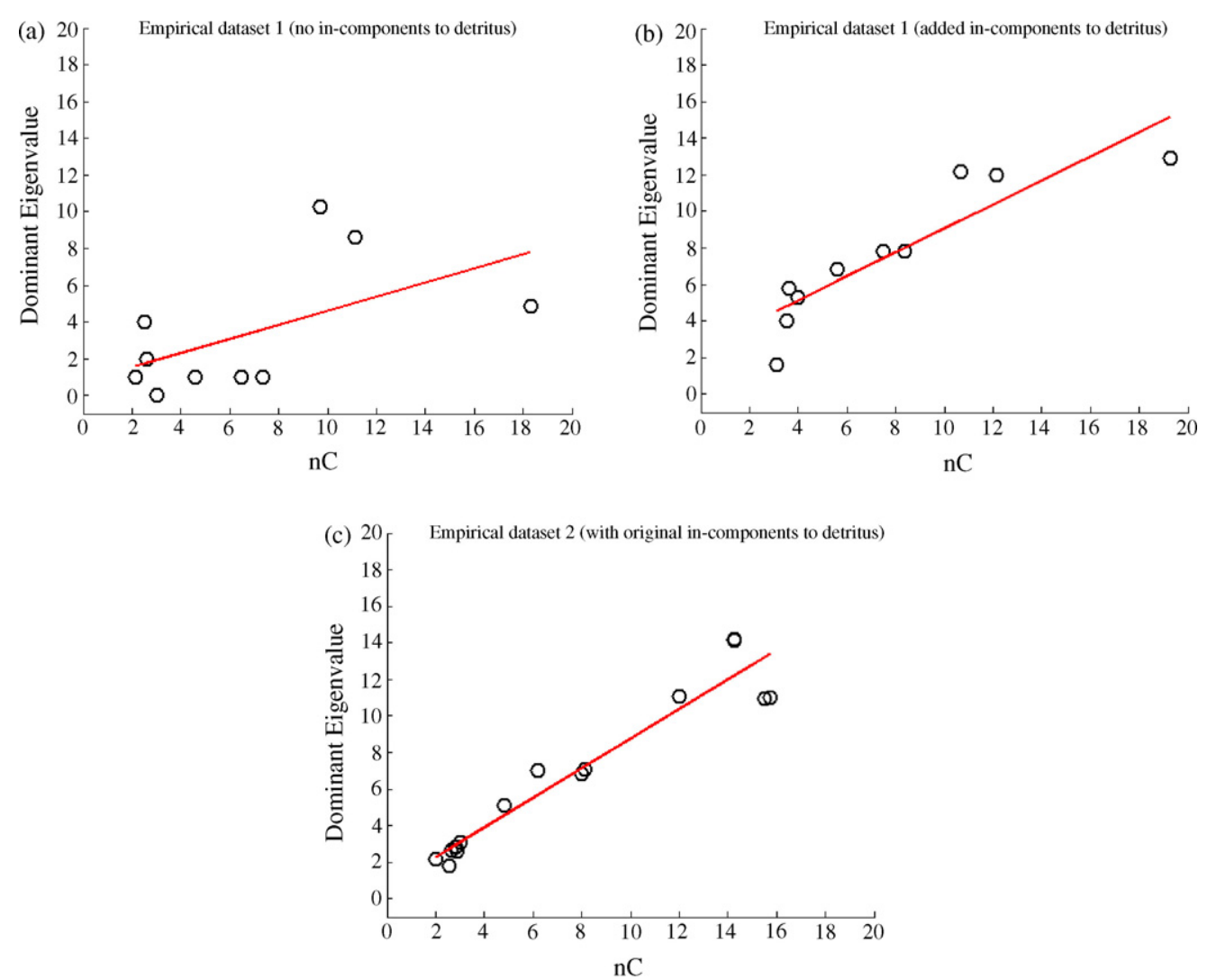

Fig. 2 - Strength of structural cycling vs. linkage density for (a) 10 empirical food webs that did not consider flows to detritus, E1 (original); $\lambda_{\max }$ ranges from 0 to 10.25; (b) 10 empirical food webs after detritus, which was present but not linked, was connected, E1 (modified); $\lambda_{\max }$ ranges from 1.62 to 12.93; (c) 16 empirical data sets, E2, which included detritus in the original model development; $\lambda_{\max }$ ranges from 1.79 to 14.17 .

tion, it is only treated as a primary food source in the structure. By reconnecting the rest of the food web to detritus such that it becomes a carrier compartment and not just a transmitter, we see that this gives a higher structural cycling value (Fig. 2b), ranging from 1.62 to 12.93. The empirical dataset, E2, that originally included detritus as a linked part of the food web includes 16 webs in which $n$ ranges from 6 to 125 (Table 1). These systems have a high cyclicity value $(\lambda \max$ ranges from 1.79 to 14.17$)$ and exhibit a similar pattern to the modified dataset above (Fig. 2c). Note that all networks with detritus included had $\lambda_{\max }>1$. These results demonstrate the high level of cyclicity present in empirically derived ecological food webs that appropriately include detrital linkages. The level of cyclicity also shows a clearer trend $\left(\lambda_{\max } \sim n C\right)$ for the data sets that include the detritus feedback.

Using each community assembly rule, we constructed a food web structure and calculated the maximum eigenvalue associated with that structure. This was repeated 1000 times to create different size $(n)$ and connectance $(C)$ structure-eigenvalue combinations for each assembly rule. A pattern emerges for each community assembly rule when looking at the maximum eigenvalue versus the linkage density, $n C=L / n$ (Fig. 3) and for simplicity we fit this pattern to a linear model. In fact, it has been shown that for large random networks $\lambda_{\max } \approx n C$ (Borrett et al., 2007), a result reproduced in our simulations. The random micro-scale interactions result in the Cyber-Ecosystem model also having a high maximum eigenvalue. The Cascade model has no structural cycling and always has a maximum eigenvalue equal to zero, while the Niche model demonstrates limited strong cycling components. Adding the detritus feedback loop (going from the Niche

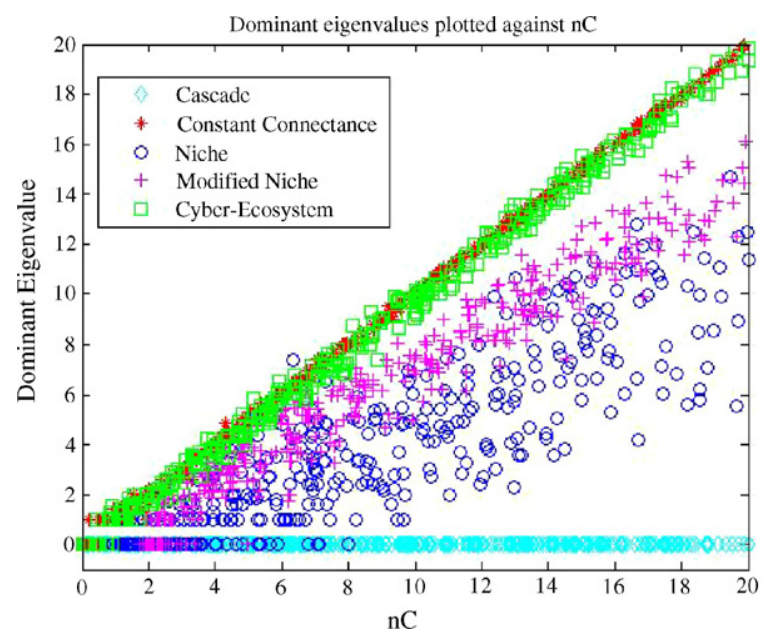

Fig. 3 - Strength of structural cycling vs. linkage density for 400 individual food web structures for each assembly rule under different size and connectance combinations. 


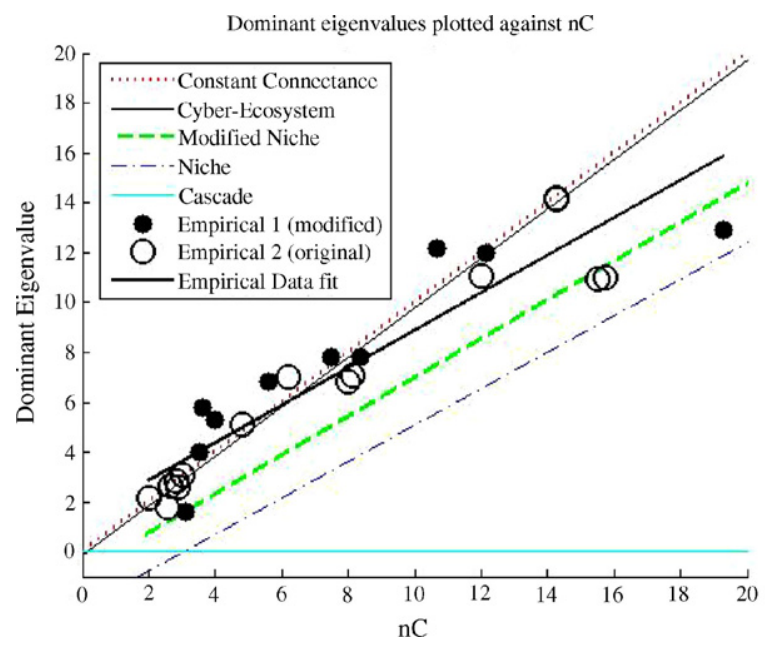

Fig. 4 - Comparison of empirical data, E1 (modified) and E2, to regression lines from five community assembly models. The regression lines were computed using 1000 runs/structure (whereof only 400 are displayed in Fig. 3 to make the plot more readable).

to the Modified Niche model) increases $\lambda_{\max }$ (cyclicity), and also decreases the variance of $\lambda_{\max }$, resulting in a clearer trend $\lambda_{\max } \sim n C$.

When we compare these models to the empirical datasets, we see that the cycling in the empirical data sets tends to lie closest to that generated by the Constant Connectance (random) and Cyber-Ecosystem models (Fig. 4). We do not contend that ecosystems are randomly connected - other structural measures show that there is a pattern of direction and articulation in ecosystems not present in random models - but the degree of cycling is closer to a Constant Connectance (random) model than the chain models that have been employed so far. The Cyber-Ecosystem model captures the high level of cycling of the Constant Connectance model but in an ecologically constrained framework. Table 2 summarizes the statistics for the analysis that led to the regression lines in Fig. 4, and shows that both the slope of $\lambda_{\max }$ (as a function of linkage density $n C$ ) and the variance in $\lambda_{\max }$ around the regression line is best fit by the Modified Niche model. However, the Modified Niche model underestimates the overall cycling observed in the empirical data particularly in smaller systems. It should be noted that some of the empirical systems have included several detritus compartments, whereas the Modified Niche model has only one, which might be one of the reasons why it underestimates $\lambda_{\max }$.

\section{Discussion}

While there are many important links in the ecological networks, in this work, we have shown that passive flows to detritus and from detritus back into the system give an important contribution to the total structural cycling in the system, and increases the trend that cycling increases with the product $n C$, both in models and empirics. Although this web-like structure was evident in Lindeman's original trophic dynamic analysis, it was lost as food webs were constructed according to a food chain paradigm that excluded the key recycling elements such as detritus and detrital feeders. The community assembly models, of course, should reflect empirics, but what precipitated was a data collection paradigm that reinforced the model's correctness. A positive feedback between model development and data collection ensued, in that researchers who expected not to find detritus links excluded them a priori from both their data collection and models, reinforcing the perception of acyclic food webs. When detritus is included as a connected compartment in the network, the structural cycling value is much higher, and this has important implications for the presence and significance of cycling. This suggests that there is significantly greater cycling present in ecological food webs than the traditional models or the uncorrected datasets reveal.

Many important consequences follow from a strong presence of structural cycling: (1) Cyclic energy pathways do exist

Table 2 - Statistics for the maximum eigenvalue $\left(\lambda_{\max }\right)$ computed from the food webs resulting from five different assembly models, and from empirical food webs with and without input to detritus

\begin{tabular}{|c|c|c|c|c|}
\hline Models & Slope & Intercept & Variance & $\lambda_{\max }$ range \\
\hline Cascade & 0.00 & 0.00 & 0.00 & $0-0$ \\
\hline Constant Connectance (CC) & 1.00 & 0.00 & 0.03 & 0-19.72 \\
\hline Niche & 0.71 & -2.00 & 5.29 & $0-14.63$ \\
\hline Modified Niche & 0.76 & -0.66 & 1.57 & $0-16.12$ \\
\hline Cyber-Ecosystem & 0.99 & -0.13 & 0.15 & 0-19.91 \\
\hline \multicolumn{5}{|l|}{ Data } \\
\hline Empirics (1) original without detritus & 0.39 & 0.76 & 8.68 & $0-10.25$ \\
\hline Empirics (1) with detritus added & 0.65 & 2.52 & 2.78 & $1.62-12.93$ \\
\hline Empirics (2) original with detritus & 0.81 & 0.68 & 1.51 & $1.79-14.17$ \\
\hline Empirics (2) with detritus removed & 0.13 & 1.12 & 0.97 & $0-3.64$ \\
\hline Empirics (1 and 2) detritus removed from (E2) & 0.23 & 0.98 & 4.53 & $0-10.25$ \\
\hline Empirics (1 and 2) detritus added to (E1) & 0.75 & 1.37 & 2.15 & $1.62-14.17$ \\
\hline
\end{tabular}

Data in italics include detritus either originally or by modification. The slope and intersection refer to the regression lines obtained by assuming a linear relation between $\lambda_{\max }$ and the linkage density $n C$. Variance refers to the variance of the data points of $\lambda$ max around the regression line. $\operatorname{Var}\left(\lambda_{\max }\right)$ does not increase with $n C$. 
in ecological food webs, and they can be identified quite easily using the eigenvalue metric presented herein. The importance of these cycles for transporting energy, at least until all energy is dissipated, is determined not only by the existence of structural cycles, but also by the transfer efficiency along each dissipative pathway, which has been investigated elsewhere in the previously cited network trophic dynamics literature. (2) While behaviorally there may be top predators, functionally there is no top as far as energy flow is concerned, only continuous dissipative paths reaching as a limit process beyond the level of $n$-trophic steps (Higashi et al., 1988; Whipple, 1998). (3) Detritus is not a disconnected, infinite-source, basal compartment but receives input from the rest of the ecological community. (4) Higashi and Patten (1989) demonstrated that cycling was one of six key network features that contributed positively indirect effects, thereby greater cycling increases the importance of indirect effects. The maximum number of cycles is $\sum_{k=1}^{n}(k-1) !\left(\begin{array}{l}n \\ k\end{array}\right)$, which gives rise to extremely large numbers of indirect pathways. Even in dissipative flow models, where long paths carry small amounts of energy, the large number of higher order pathways may give a significant contribution to the total energy flow (see Lenzen, 2007 for a quantitative analysis). (5) Allesina et al. (2005b) found that network properties are more sensitive to aggregation of the detrital components than of other components. Therefore, one must take extra care to properly represent these components. (6) Finally, with no tops to food pyramids (and no bottoms either, in strict network trophic dynamics recognizing widespread primary producer mixotrophy), the top-down and bottom-up debate regarding ecosystem control becomes even more ambiguous. As first suggested by Patten (1978), control within ecosystems is distributed with each component influencing the others (Patten and Auble, 1981; Fath, 2004b; Schramski et al., 2006).

We intend for this research to encourage other empirical and theoretical ecologists to consider the important role of detritus when collecting data and constructing models. We also commend to the attention of students of food webs the two-decades old network trophic dynamics literature we have cited, with which our present results are consistent. Network flow analysis (Patten, 1985) affords a richer picture of the role and influence of energy cycles, but often data for such a complete analysis are not available. Therefore, here we introduce a simpler, easier to implement metric to identify the presence and strength of structural cycles. These structural cycles have an impact on network parameters. There is a need in our broader field to achieve greater correspondence with the original ecosystem "food-cycle" concept introduced by Lindeman. This includes biotic and abiotic resources both, and will make explicit the true cyclic nature of ecosystem energy-matter networks. As Lindeman noted, detritus is not just a sink, but also a source for internal flows that creates cyclic energy pathways in ecosystems.

\section{Acknowledgments}

We are grateful to Stuart Borrett, and indirectly Jennifer Dunne, for providing the unmodified data set used in Fig. 2a, and to Bernie Patten, Stefano Allesina, and anonymous reviewers for comments on the manuscript. GH was a participant in IIASAs Young Scientist Summer Program and was awarded the Mikhalevich Scholarship in part for this research.

\section{REFERENCES}

Allesina, S., Bodini, A., Bondavalli, A., 2005a. Ecological subsystems via graph theory: the role of strongly connected components. Oikos 110, 164-176.

Allesina, S., Bondavalli, C., Scharler, U.M., 2005b. The consequences of the aggregation of detritus pools in ecological networks. Ecol. Model. 189, 221-232.

Borrett, S.R., Fath, B.D., Patten, B.C., 2007. Functional integration of ecological networks through pathway proliferation. J. Theor. Biol. 245, 98-111.

Burns, T.P., 1989. Lindeman's contradiction and the trophic structure of ecosystems. Ecology 70, 1355-1362.

Burns, T.P., Higashi, M., Wainright, S., Patten, B.C., 1991. Trophic unfolding of a continental shelf energy-flow network. Ecol. Model. 55, 1-26.

Christian, R.R., Luczkovich, J.J., 1999. Organizing and understanding a winter's seagrass foodweb network through effective trophic levels. Ecol. Model. 117, 99-124.

Cohen, J.E., Newman, C.M., 1985. A stochastic theory of community food webs. I. Models and aggregated data. Proc. Roy. Soc. Lond. B 224, 421-448.

Cohen, J.E., Briand, F., Newman, C.M., 1990. Community Food Webs: Data and Theory. Springer-Verlag.

Dame, R.F., Patten, B.C., 1981. Analysis of energy flows in an intertidal oyster reef. Mar. Ecol. Prog. Ser. 5, 115-124.

Dunne, J.A., Williams, R.J., Martinez, N.D., 2002. Food-web structure and network theory: the role of connectance and size. Proc. Natl. Acad. Sci. U.S.A. 99, 12917-12922.

Dunne, J.A., Williams, R.J., Martinez, N.D., 2004. Network structure and robustness of marine food webs. Mar. Ecol. Prog. Ser. 273, 291-302.

Fath, B.D., 1998. Network analysis: foundations, extensions, and applications of a systems theory of the environment. Ph.D. Thesis. University of Georgia, Athens, Georgia, 176 pp.

Fath, B.D., 2004a. Network analysis applied to large-scale cyber-ecosystems. Ecol. Model. 171, 329-337.

Fath, B.D., 2004b. Distributed control in ecological networks. Ecol. Model. 179, 235-246.

Fath, B.D., Jørgensen, S.E., Patten, B.C., Straškraba, M., 2004. Ecosystem growth and development. Biosystems 77, 213-228.

Finn, J.T., 1976. Measures of ecosystem structure and function derived from analysis of flows. J. Theor. Biol. 56, 363-380.

Goldwasser, L., Roughgarden, J., 1993. Construction and analysis of a large Caribbean food web. Ecology 74, 1216-1233.

Halnes, G., Fath, B.D., Liljenström, H., 2007. The modified niche model: including a detritus compartment in simple structural food webs. Ecol. Model. 208, 9-16.

Havens, K., 1992. Scale and structure in natural food-webs. Science 257, 1107-1109.

Heymans, J.J., Ulanowicz, R.E., Bondavalli, C., 2002. Network analysis of the South Florida Everglades graminoid marshes and comparison with nearby cypress ecosystems. Ecol. Model. 149, 5-23.

Higashi, M., Patten, B.C., 1989. Dominance of indirect causality in ecosystems. Am. Nat. 133, 288-302.

Higashi, M., Burns, T.P., Patten, B.C., 1988. Food network unfolding: an extension of trophic dynamics for application to natural ecosystems. J. Theor. Biol. 140, 243-261.

Higashi, M., Burns, T.P., Patten, B.C., 1991. Trophic niches of species and trophic structure of ecosystems: complementary 
perspectives through food network unfolding. J. Theor. Biol. 154, 57-76.

Higashi, M., Patten, B.C., Burns, T.P., 1993a. Network trophic dynamics: the modes of energy utilization in ecosystems. Ecol. Model. 66, 1-42.

Higashi, M., Burns, T.P., Patten, B.C., 1993b. Network trophic dynamics: the tempo of energy movement and availability in ecosystems. Ecol. Model. 66, 43-64.

Hill, J. Personal communication of unpublished manuscript. On largest real root of a polynomial.

Jain, S., Krishna, S., 2003. Graph theory and the evolution of autocatalytic networks. In: Bornholdt, S., Schuster, H.G. (Eds.), Handbook of Graphs and Networks: From the Genome to the Internet. Wiley-VCH, pp. 355-395.

Leguerrier, D., Niquil, N., Boileau, N., Rzeznik, J., Sauriau, P.-G., Le Moine, O., Bacher, C., 2003. Numerical analysis of the food web of an intertidal mudflat ecosystem on the Atlantic coast of France. Mar. Ecol. Prog. Ser. 246, 17-37.

Lenzen, M., 2007. Structural path analysis of ecosystem networks. Ecol. Model. 200, 334-342.

Lindeman, R.L., 1942. The trophic dynamic aspect of ecology. Ecology 23, 399-418.

Link, J., 2002. Does food web theory work for marine ecosystems? Mar. Ecol. Prog. Ser. 230, 1-9.

Martinez, N.D., 1992. Constant connectance in community food webs. Am. Nat. 139, 1208-1218.

Moore, J.C., Berlow, E.L., Coleman, D.C., de Ruiter, P.C., Dong, Q., Hastings, A., Johnson, N.C., McCann, K.S., Melville, K., Morin, P.J., Nadelhoffer, K., Rosemond, A.D., Post, D.M., Sabo, J.L., Scow, K.M., Vanni, M.J., Wall, D.H., 2004. Detritus, trophic dynamics and biodiversity. Ecol. Lett. 7, 584-600.

Opitz, S., 1996. Trophic interactions in Caribbean coral reefs. Tech Report 43, ICLARM.

Paine, R.T., 1980. Food webs: linkage, interaction strength and community infrastructure. J. Anim. Ecol. 49, 667-685.

Patricio, J., Ulanowicz, R.E., Pardal, M.A., Marques, J.C., 2004. Ascendency as an ecological indicator: a case study of the estuarine pulse eutrophiciation. Estuar. Coast. Shelf Sci. 60, 23-35.

Patten, B.C., 1978. Energy environments in ecosystems. In: Fazzolare, R.A., Smith, C.B. (Eds.), Energy Use Management, vol. IV. Pergamon Press, New York, pp. 853-857.

Patten, B.C., 1985. Energy cycling in the ecosystem. Ecol. Model. 28, 1-71.

Patten, B.C., 1988. Systems ecology of Okefenokee Swamp. In: Mitch, W.J., Straškraba, M., Jørgensen, S.E. (Eds.), Wetland Modelling. Elsevier, pp. 189-214.
Patten, B.C., Auble, G.T., 1981. System theory of the ecological niche. Am. Nat. 117, 893-922.

Patten, B.C., Higashi, M., Burns, T.P., 1990. Trophic dynamics in ecosystem networks: significance of cycles and storage. Ecol. Model. 51, 1-28.

Pimm, S.L., 2002. Food Webs. Univ. Chicago Press.

Polis, G.A., 1991. Complex trophic interactions in desserts: an empirical critique of food-web theory. Am. Nat. 138, 123-155.

Pomeroy, L.R., 1974. The ocean's food web, a changing paradigm. Bioscience 24, 499-504.

Sandberg, J., Elmgren, R., Wulff, F., 2000. Carbon flows in Baltic Sea food webs-a re-evaluation using a mass balance approach. J. Mar. Syst. 25, 249-260.

Schramski, J.R., Gattie, D.K., Patten, B.C., Borrett, S.R., Fath, B.D., Thomas, C.R., Whipple, S.J., 2006. Indirect effects and distributed control in ecosystems: distributed control in the environ networks of a seven-compartment model of nitrogen flow in the Neuse River Estuary. USA-steady-state analysis. Ecol. Model. 194, 189-201.

Seneta, E., 1973. Non-negative Matrices; An Introduction to Theory and Applications. Wiley.

Townsend, C.R., Thompson, R.M., McIntosh, A.R., Kilroy, C., Edwards, E., Scarsbrook, M.R., 1998. Disturbance, resource supply and food-web architecture in streams. Ecol. Lett. 1, 200-209.

Ulanowicz, R.E., 1983. Identifying the structure of cycling in ecosystems. Bioscience 65, 219-237.

Waide, R.B., Reagan, W.B., 1996. The Food Web of a Tropical Rainforest. Univ. Chicago Press.

Warren, P.H., 1989. Spatial and temporal variation in the structure of a fresh-water food web. Oikos 55, 299-311.

Wetzel, R.G., 1995. Death, detritus and energy flow in aquatic ecosystems. Freshw. Biol. 33, 83-89.

Whipple, S.J., 1998. Path-based network unfolding: a solution for the problem of mixed trophic and nontrophic processes in trophic dynamic analysis and nontrophic processes. J. Theor. Biol. 190, 263-276.

Whipple, S.J., 1999. Analysis of ecosystem structure and function: extended path and flow analysis of a steady-state oyster reef model. Ecol. Model. 114, 251-274.

Whipple, S.J., Patten, B.C., 1993. The problem of nontrophic processes in trophic ecology: a network unfolding solution. J. Theor. Biol. 163, 393-411.

Wiegert, R.G., Owen, D.F., 1971. Trophic structure, available resources and population density in terrestrial vs. aquatic ecosystems. J. Theor. Biol. 30, 69-81.

Williams, R.J., Martinez, N.D., 2000. Simple rules yield complex food webs. Nature 404, 180-183. 